Ann. Génét. Sél. anim., I974, 6 (2), 24I-252.

\title{
LE LOT TÉMOIN \\ DANS LE CONTRÔLE DES TAUREAUX DE RACE A VIANDE FRANÇAISE SUR DESGENDANCE CROISÉE : INTÉRET POUR LA MESURE DU PROGRES GÉNÉTIQUE
}

\author{
J. J. COLlEAU, J. L. FOULLEY et J. GAILlARD* \\ Station de Génétique quantitative et appliquée, \\ Centre national de Recherches zootechniques, I. N. R. A., \\ 78350 Jouy en Josas \\ * Institut Technique de l'Élevage bovin, Paris
}

\section{RÉSUMÉ}

Depuis I969, un lot témoin a été inclus dans les opérations normales de contrôle en ferme sur descendance croisée de taureaux de race à viande française pour la production de veaux de boucherie. Ce dispositif permet d'abord de classer des taureaux testés dans des unités de sélection différentes, suivant une méthode que nous décrivons. Il permet d'autre part de mesurer le progrès génétique réalisé dans le temps, quoiqu'il ne représente pas la seule méthode possible. Nous décrivons en effet six méthodes d'estimation du progrès génétique (coefficients de régression, moindres carrés) faisant intervenir les taureaux témoins, les taureaux de testage et les taureaux de service. Nous analysons leur précision respective dans une population théorique schématisant le cas d'une unité de sélection française. Il apparaît que l'utilisation d'un lot témoin est d'autant plus intéressante qu'on se propose de mesurer le progrès génétique réalisé sur une longue période (Io ans).

L'utilisation d'un lot témoin est prévue également pour le contrôle sur descendance en station pour la production de jeunes bovins. Elle représenterait alors la seule méthode possible de mesure du progrès génétique.

\section{I. - INTRODUCTION}

\section{A. - Historique du schéma de sélection des mâles}

Le schéma de sélection des mâles de races à viande destinés à la production de veaux de boucherie ( 3 mois) ou de jeunes bovins (I2-I 8 mois) en croisement terminal dans le Sud et le Centre de la France comporte à chaque génération trois étapes successives : 
a) production planifiée de veaux mâles à partir des meilleurs géniteurs mâles de la génération précédente ;

b) sélection de ces veaux sur performances individuelles à deux stades successifs : en ferme (poids et conformation à 3 mois) puis en station (croissance, efficacité alimentaire et conformation de 9 à $\mathrm{I} 5$ mois) ;

c) sélection sur descendance des meilleurs taurillons en ferme (veaux de boucherie abattus à 3 mois) ou en station (jeunes bovins abattus à 15 mois).

Ce schéma n'a été réalisé que progressivement de I955 à I973 par les centres d'insémination artificielle situés dans des zones dominantes de croisement industriel. L'absence initiale de population femelle de race à viande et de maîtrise de la sélection ou de la procréation des jeunes mâles explique pourquoi le développement des stades de sélection précédents a suivi un ordre inverse : chronologiquement en effet le stade $c$ a débuté de I955 à I960, le stade $b$ (contrôle individuel en station) vers I963-I965, le stade $a$ enfin ne s'est développé que vers I968-I970.

\section{B. - Conséquences sur le classement des reproducteurs après contrôle de descendance}

Les opérations de contrôle de descendance des taureaux ont un caractère ponctuel : elles sont réalisées périodiquement et indépendamment pour chaque nouvelle série de jeunes taurillons par les divers centres d'insémination intéressés. Dans la quasi totalité des cas, aucun taureau ne produit de descendants dans plusieurs séries. Chaque descendance est donc comparée à la moyenne des autres descendances de la même série contemporaine, seuls étant contrôlés les produits des taurillons mis en comparaison à l'exclusion des autres veaux nés dans les mêmes étables (VISSAC, 1964). Le regroupement des résultats de plusieurs séries réalisé par ailleurs (ANoNYME, I969) suppose l'homogénéité des valeurs génétiques moyennes des taureaux d'une série à l'autre.

Si ce point de vue était acceptable jusqu'en I963-I968, en l'absence de méthode de choix objective et de production planifiée des jeunes taurillons, la mise en œuvre des étapes $a$ et $b$ ci-dessus du schéma de sélection nécessitait de toute évidence certains ajustements : FOULLEY et GAILLARD (I973) ont mis au point un système d'indexation sur descendance faisant intervenir le niveau génétique de la série. Celui-ci est estimé à partir des pressions de sélection et des paramètres génétiques constatés au niveau du choix des parents et au niveau de la sélection sur performances individuelles.

En fait ces ajustements a priori tenant compte de pressions de sélection réalisées pour la production et la sélection des taurillons ainsi que des paramètres génétiques ne constituent que des palliatifs, la véritable solution réside dans une mesure objective de la variabilité génétique existant d'une série de taureaux à une autre pour un même centre ou pour plusieurs centres d'insémination. On utilise dans ce but un échantillon stable de taureaux dont la descendance est contrôlée à 1'occasion de chaque nouvelle série de testage de jeunes taurillons (lot témoin).

I. Le présent article est destiné à décrire les méthodes de constitution du lot témoin et d'estimation des valeurs génétiques des taurillons par comparaison des performances de leurs descendants à celles des veaux du lot témoin. 
2. A discuter l'efficacité de cette méthode pour la mesure du progrès génétique réalisé pour un centre d'insémination artificielle à la suite de l'application du schéma de sélection.

On envisagera dans chaque cas les problèmes spécifiques que posent l'application de cette méthode au cas du testage en ferme (effectif élevé de descendants par taureau et coefficient d'héritabilité faible) et à celui du testage en station (effectif faible de descendants par taureau et coefficient d'héritabilité élevé).

\section{2. - STRUCTURE DU LOT TÉMOIN E'T MÉTHODE D'INDEXATION}

La mise en place dans chaque série de testage d'un lot témoin de taureaux (depuis I 969 pour le testage en ferme) entraîne la nécessité d'un nouveau système d'indexation des reproducteurs, non plus par rapport à la moyenne de la série de taureaux contemporains (VISSAC, I964, I967), mais par rapport à la moyenne du lot témoin.

Dans la méthode actuelle, les données recueillies sur les descendances pour une zone et une année fixées sont traitées par la méthode des moindres carrés et permettent d'estimer les effets " père " séparément des effets " sexe ", " race de mère ", "format de la mère" (VISSAC, I964). La contrainte portant sur les solutions $\hat{t}_{i}$ relatives aux effets "père " est la contrainte habituelle : $\Sigma_{i} \hat{t}_{i}=0, i$ variant de I à $s$, où $s$ est le nombre de taureaux de testage dans la série. Si l'on introduit $k$ taureaux témoins supplémentaires de valeur $t_{1} \cdots t_{k}$, on peut exprimer directement la valeur des taureaux de testage $t_{k+1} \cdots t_{k+s}$ par rapport au lot témoin en posant la contrainte $\sum_{i=1}^{k} \hat{t}_{i}=0$.

L'index $\mathrm{I}_{i}$ du taureau $t_{i}$ est ensuite estimé par régression à partir des effets $\hat{t}_{i}$ en utilisant la relation $\mathrm{I}_{i}=b_{i} \hat{t}_{i}$, avec $b_{i}=\frac{\operatorname{cov}\left(\mathrm{G}_{i}, \hat{t}_{i}\right)}{v\left(\hat{t}_{i}\right)}$ ò̀ $\mathrm{G}_{i}$ désigne la valeur génétique du taureau $i$. On a alors

$$
\mathrm{I}_{i}=2 \frac{\sigma_{\mathrm{T}}^{2}}{\sigma_{\mathrm{T}}^{2}+\sigma_{e_{i}}^{2}} \hat{t}_{i}=2 \mathrm{CD}_{i} \hat{t}_{i} \quad \text { (Cunningham, I965) }
$$

où $\sigma_{\mathrm{T}}^{2}$ désigne la variance entre taureaux et $\sigma_{e_{1}}^{2}$ la variance de l'erreur d'estimation sur $\hat{t}_{i}$, variance qui peut être obtenue à partir de la variance résiduelle et de la matrice inverse de la matrice réduite des équations normalisées de moindres carrés. La précision de l'index qui correspond au rapport $\frac{\sigma_{\mathrm{T}}^{2}}{\sigma_{\mathrm{T}}^{2}+\sigma_{e_{1}}^{2}}$ et que nous nommerons coefficient de détermination $\mathrm{CD}_{i}$ dépend donc non seulement des effectifs de taureaux témoins et de testage, de la taille de leur descendance mais aussi de la distribution des effectifs selon les facteurs non génétiques. Pour simplifier la discussion, nous supposerons que les estimées $\hat{t}_{i}$ sont statistiquement indépendantes. Dans ce cas, le coefficient de détermination $\mathrm{CD}_{i}$ s'écrit

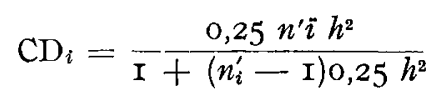

où $h^{2}$ désigne $1^{\prime}$ héritabilité du caractère considéré, $n_{i}^{\prime}$ désigne l'effectif de la descendance du taureau $i$; 
$n_{i}^{\prime}$ dépend des facteurs suivants :

$k=$ nombre de taureaux témoins

$n_{i}=$ nombre de descendants contrôlés pour le taureau $i$

$\mathrm{N}=$ moyenne harmonique du nombre de descendants par taureau dans le lot témoin

$$
n_{i}^{\prime}=\frac{n_{i} k \mathrm{~N}}{n_{i}+k \mathrm{~N}}<n_{i}
$$

La simplification adoptée est telle que l'on surestime en fait $\mathrm{CD}_{i}$ et ceci d'autant plus que la répartition des descendances par race de mère et par sexe est plus déséquilibrée.

Il apparaît d'après les relations (I) et (2) que l'introduction d'un lot témoin entraîne une baisse de précision, mais elle n'est qu'apparente car les coefficients de détermination précédemment calculés et publiés $\left(\mathrm{CD}_{i}=\frac{0,25 n_{i} h^{2}}{\mathrm{I}+\left(n_{i}-\mathrm{I}\right) 0,25 h^{2}}\right)$ étaient en fait valables intra-série, même dans le cas d'une taille de série très importante, car ils faisaient abstraction de la variance génétique entre séries, que ces séries soient échelonnées dans le temps pour un même centre ou soient issues de centres différents.

I'introduction d'un lot témoin efficient consiste à maximiser la quantité $k \mathrm{~N}$, effectif efficace du lot témoin. Il s'agit d'une part d'avoir la taille la plus grande possible pour le lot témoin, d'autre part de réduire le déséquilibre entre les descendances. En pratique, pour le premier point, le lot témoin ne devra pas avoir un effectif trop élevé car il correspond à une augmentation du coût de testage. I1 y aura donc un compromis à réaliser. En ce qui concerne le second point, la taille efficace du lot témoin s'éloignera d'autant plus de sa taille réelle qu'il y aura plus de taureaux témoins et que la probabilité d'obtention d'un veau contrôlé à partir d'une insémination de testage sera moins importante. Il faudra alors effectuer plus d'inséminations de testage pour avoir avec une probabilité donnée une taille efficace supérieure ou égale à un seuil fixé (tabl. I).

\section{TABLEAU I}

Volume d'inséminations nécessaires

à l'obtention d'un lot témoin d'effectif donné $(k N=\mathrm{I} 80)$ en fonction du nombre de taureaux $(k)$ et de la probabilité d'obtention d'un descendant contrôlé pour une IA (P)

Number of first inseminations necessary for having a control sample of a given size (I8o calves) in velation to the number of bulls $(k)$ and the probability $(P)$ of obtaining one recorded offspring from one first insemination

\begin{tabular}{|c|c|c|c|c|c|c|}
\hline \multirow{2}{*}{$\underbrace{\begin{array}{c}\text { Combinaisons } \\
k, \mathrm{~N}\end{array}}_{\text {Nombre d'IA }}$} & \multicolumn{2}{|c|}{$\begin{aligned} k & =6 \\
\mathrm{~N} & =30\end{aligned}$} & \multicolumn{2}{|c|}{$\begin{aligned} k & =9 \\
N & =20\end{aligned}$} & \multicolumn{2}{|c|}{$\begin{array}{r}k=15 \\
\mathrm{~N}=12\end{array}$} \\
\hline & $\begin{array}{c}\text { par } \\
\text { taureau }\end{array}$ & total & $\begin{array}{c}\text { par } \\
\text { taureau }\end{array}$ & total & $\begin{array}{c}\text { par } \\
\text { taureau }\end{array}$ & total \\
\hline $\mathrm{P}=0,10$ & 452 & 2712 & 340 & 3060 & 248 & 3720 \\
\hline$P=0,20$ & 221 & 1326 & 105 & 1485 & 119 & 1785 \\
\hline
\end{tabular}


Dans la situation du contrôle de descendance en ferme $\left(h^{2}=0,20 n=60\right.$ descendants par taureau), la taille efficace recherchée de l'échantillon témoin a été prise égale à I 80 . De cette manière les index sont suffisamment précis (voir tabl. 2) sans que les frais de testage soient considérablement augmentés : le total des veaux contrôlés augmenterait de 7 à $25 \mathrm{p}$. Ioo suivant les unités de sélection.

Pour une taille efficace donnée de l'échantillon témoin, il est théoriquement préférable de se limiter à un seul taureau, pour limiter le nombre des inséminations de testage. En pratique, pour des raisons psychologiques et aussi techniques, parce que les éleveurs pourraient avoir des comportements délibérés vis-à-vis de I ou 2 taureaux témoins, il est souhaitable de ne pas faire appel à un nombre de taureaux trop faible. Ainsi les lots témoins des races Charolaise, Blonde d'Aquitaine et Limousine, comprennent 8,6 et 9 taureaux respectivement, les différences entre races dépendant des décisions prises par les responsables du testage dans chacune des races. Pour que ces taureaux témoins n'entraînent pas à long terme une moins value pour les éleveurs, ils ont été choisis dans le tiers supérieur de la population des taureaux indexés.

\section{TABLEAU 2}

$V$ ariations du coefficient de détermination de l'index en fonction de l'effectif du lot témoin contrôlé en ferme et en station

The determination coefficient of the index and its variations with the size of the control sample, in farm or station progeny-testing

\begin{tabular}{c|c|c|c|c|c|c|c|c}
\hline $\begin{array}{c}\text { Effectif efficace } \\
\text { du lot témoin }\end{array}$ & 20 & 40 & 60 & 90 & 120 & 180 & 240 & 300 \\
\hline $\begin{array}{c}\text { Contrôle en ferme } \\
n=60 \\
h^{2}=0,20\end{array}$ & & & & & & & & \\
\hline $\begin{array}{c}\text { Contrôle en station } \\
n=20 \\
h^{2}=0,50\end{array}$ & 0,59 & 0,66 & 0,68 & 0,70 & 0,71 & 0,72 & & \\
\hline \hline
\end{tabular}

Les méthodes de testage sur descendance en station pour la production de jeunes bovins sont en cours d'élaboration. Dans l'hypothèse où les caractères mesurés ont alors pour héritabilité 0,50 et où on contrôle 20 descendants par taureau, 1'échantillon témoin devrait être constitué de 60 à 90 veaux (tabl. 2). Ces 60 à 90 veaux seraient alors issus au moins de 3 pères différents.

\section{3. - EFFICACITÉ DU LOT TÉMOIN POUR LA MESURE DU PROGRÈS GÉNÉTIQUE}

La taille de l'échantillon témoin en ferme (I80 veaux croisés) a été déterminée principalement de manière à assurer un classement suffisamment précis des taureaux sans alourdir excessivement les frais de contrôle de descendance. Il est par ailleurs 
important de savoir si l'utilisation d'un tel lot témoin est efficace pour la mesure du progrès génétique et de situer cette efficacité par rapport à celle d'autres méthodes.

\section{I. - DESCRIPTION DES MÉTHODES COMPARÉES}

D'une façon générale on peut mesurer le progrès génétique :

- soit à partir de l'information relative aux séries indépendantes de taurillons mis au testage périodiquement : on mesure alors le progrès génétique potentiel ;

- soit à partir de l'information concernant les descendants des taureaux de service favorablement testés. Un contrôle massal, simplifié par rapport au précédent est réalisé de façon systématique et continue dans le temps dans un certain nombre de fermes. On mesure dans ce cas le progrès génétique réel lié à 1'utilisation des taureaux testés par les éleveurs.

Dans chacune de ces 2 situations le témoin génétique stable peut être :

- soit un lot de taureaux constant dans le temps sur une période longue (5 à Io ans) ;

- soit les taureaux de service favorablement testés qui sont utilisés au moins deux années consécutives.

Ces diverses possibilités d'estimation du progrès génétique vis-à-vis de la nature et de la structure des échantillons des animaux ont été schématisées sur la figure $\mathrm{I}$.

Nous allons étudier les méthodes d'estimation du progrès génétique utilisables dans chacune de ces situations :

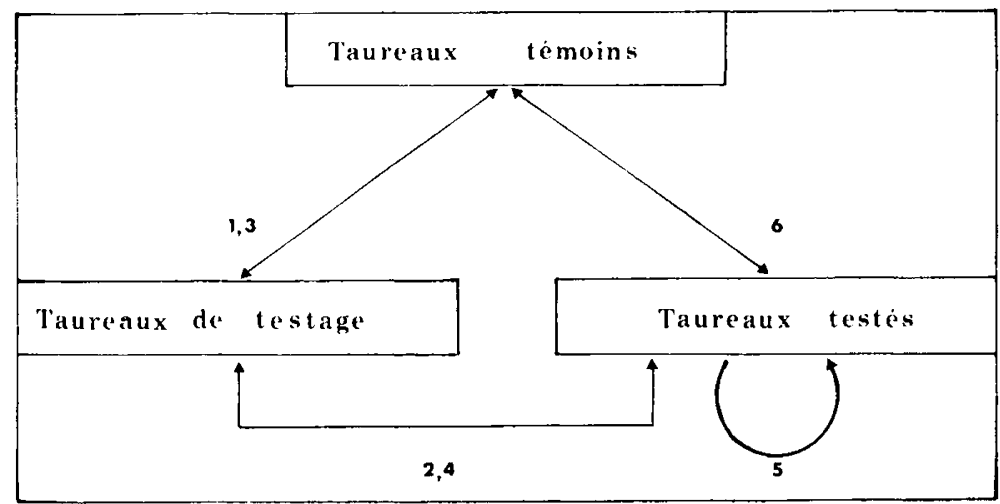

FIG. I. - Désignation des méthodes d'estimation du progrès génétique suivant la source d'information utilisée

Taureaux de testage: taurillons contrôlés sur descendance sur une période de temps limitée.

Taureaux testés : taureaux de service du centre utilisés de manière non planifiée.

Taureaux témoins : échantillon de taureaux contrôlés dans les mêmes conditions que les taureaux de testage et stables dans le temps (5 ou ro ans).

\section{A. - Méthodes utilisant les régressions}

Nous en énumérons 4 qui s'inspirent toutes de celles décrites par Smith (I962) en faisant intervenir soit des différences de coefficients de régression (méthodes I et 2) soit des coefficients de régression de déviations (méthodes 3 et 4 ). 


\section{A. I. Différences de coefficient de régression.}

\section{Méthode 1 (série de testage + lot témoin).}

On suppose que le niveau génétique des taureaux utilisés en croisement augmente linéairement de $2 \Delta \mathrm{G}$ par an et que le progrès annuel non génétique est également linéaire de valeur $\Delta \mathrm{E}$. Le coefficient de régression $b_{1}$ des performances des descendants issus des séries de testage en fonction de l'année a pour espérance mathématique $\Delta \mathrm{G}+\Delta \mathrm{E}: \mathrm{E}\left(b_{1}\right)=\Delta \mathrm{G}+\Delta \mathrm{E}$. Par ailleurs, si l'on régresse en fonction de l'année, les performances des descendants issus du lot témoin $\left(b_{2}\right)$, ce coefficient de régression a pour espérance $\Delta \mathrm{E}, \mathrm{E}\left(b_{2}\right)=\Delta \mathrm{E}$. Un premier estimateur de $\Delta \mathrm{G}$ est donc donné par $b_{1}-b_{2}$ puisque $\mathrm{E}\left(b_{1}-b_{2}\right)=\Delta \mathrm{G}$. Cette conclusion est valable quelque soit le niveau de progrès génétique réalisé sur les mères (de races laitière ou rustique). Si ce progrès est $2 \mathrm{dg}$ par exemple on a en effet $\mathrm{E}\left(b_{1}\right)=\Delta \mathrm{G}+\mathrm{dg}+\Delta \mathrm{E}$ et $\mathrm{E}\left(b_{2}\right)=\mathrm{d} g+\Delta \mathrm{E}$. La variance d'échantillonnage de $\Delta \mathrm{G}$ est calculée à partir de celle de coefficients de régression $b_{1}$ et $b_{2}$.

\section{Méthode 2 (séries de testage + taureaux testés).}

On calcule également le coefficient de régression $b_{1}$. Mais $\Delta \mathrm{E}_{1}$ est estimé grâce au coefficient de régression sur l'année $b_{3}$ des performances intra taureaux de service. Cela suppose en toute rigueur l'identité de $\Delta \mathrm{E}$ dans les deux catégories d'élevages concernés (élevages pratiquant le testage ou utilisant des taureaux testés). On suppose aussi que l'évolution des performances de la descendance suivant l'année est la même quel que soit le taureau considéré. Or en pratique, certains taureaux de service auront tendance à être utilisés différemment suivant le type de veaux produits et la race de mère considérée. L'utilisation d'une telle méthode sur des données réelles conduirait donc peut-être à des ajustements statistiques préalables.

\section{A. 2. Coefficients de régression de déviations.}

Méthode 3 (séries de testage + lot témoin).

Dans le cas où l'on dispose de taureaux témoiris, la déviation entre les performances des séries de testage et les performances de l'échantillon témoin $d_{i}=x_{i}-\mathrm{X}_{i}$ est corrigée pour les effets de milieu. L,e coefficient de régression de $d_{i}$ en fonction de $i$ numéro de l'année $\left(b_{4}\right)$ est donc une estimée de $\Delta \mathrm{G}$. L'intérêt de cette méthode est qu'elle ne suppose pas une tendance linéaire de l'effet année indépendant de la valeur génétique des taureaux.

\section{Méthode 4 (séries de testage + taureaux testés).}

Les taureaux de service peuvent être comparés année par année aux taureaux de testage des années correspondantes sous forme de déviations qui sont alors indépendantes des effets de milieu. En présence d'un progrès génétique $\Delta \mathrm{G}$ sur les veaux croisés, l'espérance du coefficient de régression intra taureau $b_{5}$ de ces déviations est égale à $-\Delta \mathrm{G}$. Cela suppose que les différences non génétiques entre les deux types d'élevages utilisant des taureaux en testage ou des taureaux de service ne varient pas dans le temps. 


\section{B. - Méthodes utilisant les moindres carrés}

Ces méthodes visent à estimer simultanément les effets " année "(non nécessairement linéaires) et " série " de taureaux contemporains (mis au testage simultanément). Dans le cas où l'effectif de la série est très élevé, cette série est très bien échantillonnée et on peut appliquer la méthode des moindres carrés, les effets " année " et " série " étant fixés, comme le proposent Hickman et Frekman (I969). Dans le cas contraire, on améliore la précision des estimations des effets " série " en considérant l'effet " série " comme un facteur aléatoire. Cela revient alors à utiliser le modèle mixte posé par HENDERson et al. (1959) et dont la résolution théorique est décrite par SEARLE (I97I). Cette méthode des moindres carrés généralisés correspond à la méthode du maximum de vraisemblance dans le cas où les facteurs aléatoires suivent une loi normale et où les composantes de la variance sont exactement connues.

\section{Méthode 5 (taureaux testés).}

Les performances des seuls taureaux testés sont analysées suivant un modèle qui tient compte de l'effet année (fixé) de l'effet linéaire du numéro de série (fixé) et de l'effet taureau intra année de naissance (aléatoire). Le deuxième effet correspond à $\Delta \mathrm{G}$. Pour l'estimer, on pose la matrice des moindres carrés habituelle mais en modifiant la diagonale de la sous-matrice correspondant à l'effet " taureau " (SEARLE, I97I). Si M est cette matrice modifiée, on peut montrer que la variance de $\Delta \mathrm{G}$ est égale au terme de $\mathrm{M}^{-1}$ correspondant au deuxième effet, multiplié par la variance d'erreur sur une série une année donnée.

Méthode 6 (taureaux testés + lot témoin).

Les performances de la descendance d'un taureau une année donnée sont ramenées en déviations par rapport à la moyenne du lot témoin. Ces déviations sont ensuite analysées suivant un modèle mixte où les effets " année " et " taureau intra année de naissance "sont aléatoires et où l'effet linéaire du numéro de série est fixé, ce dernier correspondant alors à $\Delta \mathrm{G}$.

\section{2. - RÉsultats ÉT Discussion}

Nous avons simulé le cas d'une importante unité de sélection française et utilisé les paramètres relatifs à cette unité pour comparer la précision d'estimation du progrès génétique par les méthode ci-dessus.

\section{A. - Paramètres utilisés}

a) Paramètres fixés.

- la taille de l'échantillon témoin : I80 veaux par an en ferme (voir ci-dessus)

60 veaux par an en station ;

- le nombre annuel de veaux contrôlés :

par taureau de testage : 60 en ferme et 20 en station.

par taureau testé : 20 en ferme;

- l'effectif total de la taurellerie : 30 ;

- le taux de sélection après le testage : I taureau sur 2. 
b) Paramètres variables. génétique.

- la durée $A$ de la période au cours de laquelle on désire mesurer le progrès

On supposera dans le cas des méthodes faisant intervenir les taureaux de service que l'on ne dispose pas de données exploitables avant la période considérée. On prendra $A=5$ et Io.

- la durée supposée constante d'utilisation des taureaux testés. On prendra $a=3$ et 5 ; la valeur de 5 représentant plutôt les conditions actuelles et la valeur de 3 les conditions futures. Le nombre annuel de nouveaux taureaux testés est ainsi égal à $\frac{30}{a}$ et le nombre annuel de taureaux en testage est égal à $\frac{60}{a}$.

B. - Précision de l'estimation du progrès génétique (tabl. 3)

TABLEAU 3

Écart-type d'échantillonnage du progrès génétique annuel sur les descendants croisés mesuré en millièmes d'écart-type intra-père intra-année, selon la méthode d'estimation, la durée de la période d'étude (A) et la durée d'utilisation des taureaux testés (a)

Random standard errors of the yearly genetic change on crossbred progeny according to the estimation procedure, the length of the period studied (A) and the length of utilisation of progeny-tested bulls $(\boldsymbol{v})$

The unit is $10^{-3}$ within sire-within year standard error

\begin{tabular}{|c|c|c|c|c|c|c|}
\hline \multirow{2}{*}{ Lieu } & \multirow{2}{*}{ Méthode } & \multirow{2}{*}{ No de la méthode } & \multicolumn{2}{|c|}{$A=5$ ans } & \multicolumn{2}{|c|}{$A=10$ ans } \\
\hline & & & $a=3$ ans & $a=5$ ans & $a=3$ ans & $a=5$ ans \\
\hline \multirow{5}{*}{$\begin{array}{c}\text { Ferme } \\
\text { Veaux de } \\
\text { boucherie } \\
\left(h^{2}=0,2\right)\end{array}$} & \multirow{3}{*}{ régressions } & $\begin{array}{c}1 \text { et } 3(1) \\
\text { (séries de testage } \\
+ \text { lot témoin) }\end{array}$ & 30 & 33 & 11 & 12 \\
\hline & & $\begin{array}{c}2 \\
\text { (séries de testage } \\
+ \text { taureaux testés) }\end{array}$ & 33 & 32 & 18 & 14 \\
\hline & & $\begin{array}{c}4 \\
\text { (séries de testage } \\
+ \text { taureaux testés) }\end{array}$ & 29 & 23 & 18 & 12 \\
\hline & \multirow{2}{*}{$\begin{array}{l}\text { moindres } \\
\text { carrés }\end{array}$} & $\begin{array}{c}\mathbf{5} \\
\text { (taureaux testés) }\end{array}$ & 26 & 18 & 17 & 11 \\
\hline & & $\begin{array}{c}6 \\
\text { (taureaux testés } \\
+ \text { lot témoin) }\end{array}$ & 22 & 17 & 10 & 10 \\
\hline $\begin{array}{c}\text { Station } \\
\text { jeunes bovins } \\
\left(h^{2}=0,5\right)\end{array}$ & régressions & $\begin{array}{c}1 \text { et } 3\left(^{1}\right) \\
\text { (séries de testage } \\
+ \text { lot témoin) }\end{array}$ & 50 & 56 & 18 & 19 \\
\hline
\end{tabular}

(1) Dans notre cas particulier où les effectifs sont équilibrés suivant les taureaux et suivant les années, les méthodes 1 et 3 sont équivalentes. 
a) Suivant la source d'information.

Sur des périodes courtes de mesure du progrès génétique ( 5 ans) les méthodes utilisant les descendances de taureaux testés seuls (méthode 5) ou comme étalon génétique stable (méthodes 2 et 4 ) sont plus précises que celles basées sur l'emploi d'un lot de taureaux témoins et de descendances de séries de jeunes taurillons. Sur de longues périodes (Io ans) c'est en général le contraire.

\section{b) Suivant la méthode statistique d'estimation.}

Les différences entre les coefficients de régression (méthodes I et 2) ne sont jamais plus précises que les coefficients de régression des déviations sur le temps (méthodes 3 et 4 ).

La méthode des moindres carrés est la méthode la plus efficace pour utiliser l'information provenant des taureaux de service.

c) Suivant le type de production et les modalités de contrôle des produits de testage.

La précision d'estimation du progrès génétique est netterhent supérieure dans le cas des veaux de boucherie ; en raison en grande partie de la taille de l'échantillon témoin correspondant.

\section{C. - Utilisation pratique}

a) Mise en évidence du progrès génétique.

Avec les paramètres phénotypiques des poids à 75 jours et à 400 jours calculés par Poujardieu et Vissac (I968), Frebling, Gailitard et Vissac (I972), il devrait être possible sur 5 ans de détecter au seuil de $5 \mathrm{p}$. Ioo un progrès génétique annuel de $0,5 \mathrm{p}$. Ioo pour les veaux de boucherie et de $0,7 \mathrm{p}$. Ioo pour les jeunes bovins. Sur Io ans les valeurs correspondantes seraient de 0,2 p. Ioo dans les deux cas. Le progrès génétique devrait pouvoir être détecté puisque les schémas de sélection actuels conduisent à un progrès génétique attendu de $0,8 \mathrm{p}$. Ioo pour le poids des veaux à 75 jours et de $0,6 \mathrm{p}$. Ioo pour le poids des taurillons à 400 jours.

b) Comparaison des progrès génétiques effectués par des centres de même taille.

L'écart devrait être très important pour pouvoir être détecté sur 5 ans : 0,7 p. Ioo et I p. Ioo pour les veaux et les taurillons respectivement. Sur une période de ro ans, ces valeurs s'abaissent à $0,25 \mathrm{p}$. Ioo. Dans le cas des jeunes bovins par exemple, cela veut dire que l'on pourrait discriminer un centre où le progrès génétique est de 0,60 p. Ioo d'un autre où il est de 0,35 p. Ioo. De tels écarts sont sans doute improbables.

\section{IV. - PERSPECTIVES}

Cette note précise en définitive les conditions d'emploi d'un lot témoin stable de taureaux dans le cadre des opérations de contrôle de descendance des mâles de races à viande. Cette méthode appliquée depuis I 969 dans les centres français prati- 
quant le contrôle de veaux de boucherie en ferme est actuellement (I973) en cours de mise en place pour le contrôle des descendances (taurillons) en station.

La précision d'estimation du progrès génétique par cette méthode comparée aux autres systèmes utilisables (évolution entre taureaux et intra taureaux avec le temps des descendances de taureaux de service) apparaît surtout sur une longue période (au-delà de 5 ans). Toutefois les comparaisons effectuées entre les diverses méthodes dans le cas des unités de sélection françaises présentent des fluctuations telles qu'il serait utile de les reprendre pour vérifier leur adaptation à d'autres situations. On doit néanmoins insister sur l'intérêt complémentaire qu'elle présente pour comparer le niveau génétique des mâles contemporains utilisés par plusieurs unités de sélection : cette comparaison suppose bien entendu que les échantillons de femelles supports (âge, race et type) soient comparables dans les divers centres.

Il serait évidemment souhaitable, dans la mesure où les contraintes sanitaires et économiques le permettent, que l'utilisation de cette méthode aux races à viande françaises puisse dépasser le cadre national et intéresser les opérations de sélection et de contrôle de descendance entreprises par d'autres pays sur des échantillons d'animaux de ces races (Charolaise, Limousine notamment).

Reçu pour publication en février 1974.

\title{
SUMMARY
}

\author{
MEASUREMENT OF GENETIC CHANGE BY UTILIZATION \\ OF A CONTROL SAMPLE IN BULLS TESTED ON CROSSBRED PROGENY \\ IN FRENCH BEEF BREEDS
}

Since 1969, a control sample has been included into the current farm testings on crossbred progeny of bulls from French beef breeds, for the production of veal calves. This procedure is set up to classify the bulls tested in different selection units according to a method described in this paper. It can also be used to measure the genetic change realized in the course of time, but it does not represent the only technique available. Six different methods using control bulls, young bulls and current bulls (regression coefficient, least squares) are described for estimating genetic change. Their respective accuracy have been analysed in a theoretical population, schematizing the case of a French selection unit. It appears that the utilization of a control sample is all the more efficient as the measurement concerns the genetic change realized over a long period (Io years).

Control samples are also planned to be used in station progeny-testing for baby-beef production. This method would then be the only one allowing measurement of genetic change.

\section{RÉFÉRENCES BIBLIOGRAPHIQUES}

Anonyme, 1969. Taureaux indexés. Races à viande. C. N. R.Z., I. T.E. B., Paris.

Cunningham E. P., r965. The evaluation of sires from progeny test data. Anim. Prod. 7, $221-23$ I. Foulley J. L., Gaillard J., I973. Méthode d'indexation des taureaux pour la production de viande. Ann. Génét. Sél. anim. (à paraître).

Frebling J., Gaillard J., Vissac B., I972. Mise en place et efficacité du schéma de sélection des taureaux de race à viande pour le croisement industriel. Bull. tech. Dep. Génét. anim. (I. N. R. A., Fr.) $n^{\circ} \mathbf{r} 5$.

Henderson C. R., Kempthorne O., Searle S. R., Von Krosigh C. M., I959. The estimation of environmental and genetic trends from records subjects to culling. Biometrics 15, 192-218. 
Hickman C. G., Freeman A. E., I969. New approach to experimental designs for selection studies in dairy cattle and other species. J. Dairy Si, 52, ro44-1054.

Poujardieu B., Vissac B., I968. Étude biométrique de la valeur bouchère des veaux croisés Charolais et Limousin. I. Paramètres génétiques et phénotypiques. Ann. Zootech., 17, 143-158.

SeARle S. R., I97I. Topics in variance compoment estimation. Biometrics 27, I, $1-76$.

SeArle S. R., I97r. Linear models. John Wiley. New York.

Sмгтн C., I962. Estimation of genetic change in farm livestock using field records. Anim. Prod. 4, $239-25 I$.

Vissac B., I964. Méthode de détermination de l'index génotypique des taureaux d'insémination sur la valeur de leurs veaux de boucherie. Ann. Zootech., 13, 267-275.

Vissac B., 1967. Note relative à la comparaison des descendances de taureaux dans le cas d'épreuves discontinues. Ann. Zootech., 16, 335-34r. 